\title{
ON A PROBLEM OF RANKIN ABOUT THE EPSTEIN ZETA-FUNCTION
}

\author{
by J. W. S. CASSELS
}

(Received 29th December, 1958)

1. Introduction. Let

$$
h(m, n)=\alpha m^{2}+2 \chi m n+\beta n^{2}
$$

be a positive definite quadratic form with determinant $\alpha \beta-\chi^{2}=1$. A special form of this kind is

$$
Q(m, n)=2 \cdot 3^{-\sharp}\left(m^{2}+m n+n^{2}\right) .
$$

We consider the Epstein zeta-function

$$
Z_{h}(s)=\sum_{\substack{m, n \text { integers } \\ \text { not } m=n=0}}\{h(m, n)\}^{-s},
$$

the series converging for $s>1$. For $s \geqslant 1.035$ Rankin [1] proved the following

Statement R.

$$
Z_{h}(s)-Z_{Q}(s) \geqslant 0
$$

The sign of equality is needed only when $h$ is equivalent to $Q$.

When $s$ is large, this statement suggests itself, since $Z_{h}(s)$ is dominated by those integer pairs $(m, n)$ for which $h(m, n)$ is smallest, and the forms equivalent to $Q(m, n)$ are well known to be precisely the unimodular forms $h$ for which

$$
\min _{(m, n) \neq(0,0)} h(m, n)
$$

is greatest. It is perhaps rather surprising that the statement $R$ continues to hold so far as $s=1.035$, and Rankin asked if it continued to hold up to $s=1$. In this note we shall show that this is the case and indeed rather more. The function $Z_{h}(s)$ may be analytically continued over the whole plane. Its only singularity is at $s=1$, where it has a pole with residue $\pi$. We shall prove the following theorem :

Theorem. The statement $\mathrm{R}$ holds for all $s \geqslant 0$.

We note that the statement $\mathrm{R}$ is meaningful even for $s=1$, since $Z_{h}(s)-Z_{Q}(s)$ is regular there. This case has indeed a special interest since it is connected with the Kronecker Limit Theorem which plays a part in the old-fashioned treatment of modular functions (cf. Weber [3]; for an interesting application see Kronecker [6]). We shall, however, assume that $s \neq 1$ and leave to the reader the trivial modifications required to deal with $s=1$.

For $s \leqslant 0$ it is easy to see how the statement $\mathrm{R}$ should be modified, since $Z_{h}(s)$ satisfies the functional equation.

(cf. Deuring [3]).

$$
\pi^{-s} \Gamma(s) Z_{h}(s)=\pi^{s-1} \Gamma(1-s) Z_{h}(1-s)
$$

Our proof is a slight modification of Rankin's but we give incidentally a simplification in part of the range considered by him. When $s \geqslant 3$, Rankin gave an elementary proof on 
quite different lines from his proof for $1 \cdot 035 \leqslant s \leqslant 3$. As our proof here does not work, at least without modification, for large $s$, we shall consider only the case

$$
0 \leqslant s \leqslant 3 .
$$

I am grateful to Professor Rankin for suggesting improvements and corrections to the first draft.

2. Preliminaries. Since $h(m, n)$ has unit determinant, it may be put in the shape

with $y>0$. We write

$$
h(m, n)=y^{-1}\left\{(m+n x)^{2}+n^{2} y^{2}\right\}
$$

$$
Z_{h}(s)=G(x, y)(s),
$$

and omit the $(s)$ if it does not cause confusion. Put

$$
z=x+i y \text {. }
$$

Then, for fixed $s$, the function $G(x, y)$ is invariant under the substitutions of the modular group acting on $z:$ it is not a modular function of $z$ in the usual sense since it is not analytic. On developing $G(x, y)$ as a Fourier expansion for $x$, one obtains for $s>1$ the expansion

$$
\begin{aligned}
G(x, y)=2 y^{s} \zeta(2 s)+2 y^{1-s} \zeta(2 s-1) \Gamma\left(\frac{1}{2}\right) \Gamma\left(s-\frac{1}{2}\right) / \Gamma(s) \\
+\frac{8 \pi^{s} y^{\ddagger}}{\Gamma(s)} \sum_{r>0} r^{s-1} \sigma_{1-2 s}(r) K_{s-\downarrow}(2 \pi r y) \cos 2 \pi r x,
\end{aligned}
$$

where

$$
\sigma_{k}(n)=\sum_{d \mid n} d^{k}
$$

and

$$
\begin{aligned}
K_{v}(u) & =\int_{0}^{\infty} e^{-u \cosh t} \cosh v t d t \\
& =\frac{\Gamma\left(\nu+\frac{1}{2}\right) 2^{\nu}}{u^{\nu} \Gamma\left(\frac{1}{2}\right)} \int_{0}^{\infty} \frac{\cos x u}{\left(x^{2}+1\right)^{\nu+1}} d x
\end{aligned}
$$

is a Bessel function [ef. Rankin's paper, and Watson $[4, \S 6.3]$ for the equality of the two integrals for $K_{v}(u)$. The second, which is valid only when $\nu>\frac{1}{2}$, is the one which naturally arises in the development of $G(x, y)$ in a Fourier series. The first integral, which is valid for all $\nu$ provided that $R u>0$, is the one which will be used in the sequel, as it was by Rankin.]

On applying the functional equation for the Riemann $\zeta$-function to the second term, one obtains

$$
\frac{1}{2} \Gamma(s) \pi^{-s} G(x, y)=\phi(s)+\phi(1-s)+4 y^{1} \sum_{r \geqslant 1} r^{s-\frac{1}{3}} \sigma_{1-2 s}(r) K_{s-\frac{1}{t}}(2 \pi r y) \cos 2 \pi r x,
$$

where

$$
\phi(s)=(y / \pi)^{s} \Gamma(s) \zeta(2 s) .
$$

This gives us the continuation of $G(x, y)(s)$ to the whole $s$-plane. Incidentally, since $K_{\nu}(u)=K_{-\nu}(u)$, it also gives the functional equation (2). 
3. Outline of Proof. In $\S \S 4,5$ we shall prove the following two lemmas about the partial derivatives of $G(x, y)$ with respect to $x$ and $y$.

LEMMa 1. $G_{\nu}(x, y)>0$ for $y \geqslant \frac{3}{2}$.

LEMMa 2. $G_{x}(x, y)<0$ for $y \geqslant \frac{3}{5}$ and $0<x<\frac{1}{2}$.

Both of these lemmas play a part in Rankin's paper for one of the ranges $(1 \cdot 035 \leqslant s \leqslant 2)$ considered by him.

For the sake of completeness we reproduce Rankin's argument showing that Statement $R$ follows from Lemmas 1 and 2.

When the form $h(m, n)$ is reduced, $(x, y)$ lies in the modular region

$$
D: \quad 0 \leqslant x \leqslant \frac{1}{2}, \quad y>0, x^{2}+y^{2} \geqslant 1 \text {. }
$$

Since $Q(x, y)$ is a continuous function, it must, by Lemma 1 , attain its minimum at some point $\left(x^{\prime}, y^{\prime}\right) \in D$ with $y^{\prime}<\frac{3}{2}$. By Lemma 2, we must have $x^{\prime}=\frac{1}{2}$. But now

$$
G\left(x^{\prime}, y^{\prime}\right)=G\left(x^{\prime \prime}, y^{\prime \prime}\right)
$$

where

so that

$$
x^{\prime \prime}+i y^{\prime \prime}=\frac{1}{1-\left(x^{\prime}+i y^{\prime}\right)}=\frac{2}{4 y^{\prime 2}+1}+i \frac{4 y^{\prime}}{4 y^{\prime 2}+1}
$$

$$
0<x^{\prime \prime} \leqslant \frac{1}{2}, \quad y^{\prime \prime} \geqslant \frac{3}{5},
$$

since $3^{\ddagger} / 2 \leqslant y^{\prime} \leqslant \frac{3}{2}$. By Lemma 2 , we must have $x^{\prime \prime}=\frac{1}{2}$. Hence $y^{\prime}=3^{\natural} / 2$. That is, in the modular region $D$ the function $G(x, y)$ attains its minimum at $x=\frac{1}{2}, y=3^{\ddagger} / 2$, and only there. This is just statement $R$.

In the rest of this note we shall prove Lemmas 1 and 2 by differentiating the identity of $\S 2$, and estimating the resulting expressions.

4. Proof of Lemma 2. On differentiating the identity (4) for $G(x, y)$ term by term we obtain

$$
G_{x}(x, y)=-\frac{16 \pi^{s+1} y^{\frac{2}{2}}}{\Gamma(s)} \Lambda
$$

where we have written

$$
\Lambda=\sum_{r \geqslant 1} r^{s+\sigma_{1-2 s}}(r) K_{s-\frac{1}{\mathrm{~d}}}(2 \pi r y) \sin 2 \pi r x .
$$

On substituting the integral $\left(5_{1}\right)$ for $K_{s-t}(2 \pi r y)$ and interchanging summation and integration we obtain

$$
\Lambda=\int_{0}^{\infty} \psi\left(\delta_{t}\right) \cosh \left(s-\frac{1}{2}\right) t d t
$$

where

$$
\delta_{t}=e^{-2 \pi y \cosh ^{\prime} t}
$$

and 


$$
\psi=\psi(\delta)=\sum_{r \geqslant 1} r^{s+1} \sigma_{1-2 s}(r) \delta^{r} \sin 2 \pi r x .
$$

We note that

$$
\delta_{t} \leqslant e^{-2 \pi y} \leqslant e^{-6 \pi / 5}<40^{-1},
$$

since $y \geqslant 3 / 5$. Hence it will be enough to show that

whenever

$$
\psi(\delta)>0
$$

$$
\left.\begin{array}{l}
0<x<\frac{1}{2} \\
0<\delta<40^{-1} \\
0 \leqslant s \leqslant 3
\end{array}\right\}
$$

In (7) we have

$$
\sigma_{1-2 s}(r)=\sum_{d \mid r} d^{1-2 s}
$$

Put $r=d f$ and change the order of summation in (7). Then we have

where

$$
\psi=\sum_{d \geqslant 1} d^{n}-s \omega_{d}
$$

$$
\omega_{d}=\sum_{f \geqslant 1} f^{s+\frac{1}{3}} \delta^{d f} \sin 2 \pi d f x .
$$

We now obtain various estimates for $\omega_{d}$. In the first place, quite trivially,

$$
\begin{aligned}
\left|\omega_{d}\right| & \leqslant \sum_{f} \geqslant 1 \\
& \leqslant \frac{\delta^{s+1} \delta^{d f}}{\left(1-\delta^{d}\right)^{16}}, \ldots \sum_{f \geqslant 1} f^{4} \delta^{d f}
\end{aligned}
$$

the last inequality holding because the expansion of the last line majorizes the previous line,

On applying partial summation following Rankin, one also obtains

$$
4 \sin ^{2}(\pi d x) \omega_{d}=\sum_{f \geqslant 1} g_{f}\{(f+1) \sin 2 \pi d x-\sin 2 \pi(f+1) d x\},
$$

where

$$
\begin{aligned}
g_{f} & =f^{s+1} \delta^{d f}-2(f+1)^{s+1} \delta^{d(f+1)}+(f+2)^{s+1} \delta^{d(f+2)} \\
& \geqslant f^{s+1} \delta^{d f}\left\{1-2[(f+1) / f]^{s+1} \delta^{d}\right\} \\
& \geqslant f^{s+1} \delta^{d f}\left(1-2^{5} \delta^{d}\right) \\
& >0
\end{aligned}
$$

by (9). We deduce from (11) that

for all $d$ such that

$$
\omega_{d}>0
$$

$$
0<d x<\frac{1}{2}
$$

By hypothesis, (13) is true with $d=1$. Since $x>0$, there is a greatest $d$, say $d_{0}$, such that (13) holds, so that

Then, by (11),

$$
\frac{1}{4} \leqslant d_{0} x<\frac{1}{2} .
$$




$$
\begin{aligned}
4 \omega_{d_{0}} & \geqslant 4 \sin ^{2}\left(\pi d_{0} x\right) \omega_{d_{0}} \\
& =\sum_{f \geqslant 1} g_{f}\left\{(f+1) \sin 2 \pi d_{0} x-\sin 2 \pi(f+1) d_{0} x\right\} \\
& \geqslant \sum_{f \geqslant 1} g_{f}\left\{(f+1) 2^{-\downarrow}-1\right\} \\
& =\left(2^{\downarrow}-1\right) \delta^{d_{0}}+\left(1-2^{-\downarrow}\right) 2^{s+1} \delta^{2 d_{0}},
\end{aligned}
$$

on substituting the values (12) for $g_{f}$ and arranging in powers of $\delta$. Hence

$$
\omega_{d 0} \geqslant \frac{1}{4}\left(2^{\frac{1}{2}}-1\right) \delta^{d_{0}} \text {. }
$$

Since $\omega_{d}>0$ for $d<d_{0}$, we deduce that

Hence

$$
\psi \geqslant \sum_{d \geqslant d_{0}} d i-8
$$

$$
d_{0}^{8-\frac{d}{2}} \delta^{-d_{0}} \psi \geqslant \frac{1}{4}\left(2^{\frac{d}{d}}-1\right)-\sum_{d>d_{0}}\left(\frac{d}{d_{0}}\right)^{\frac{1}{2}-s} \frac{\delta^{d-d_{0}}}{\left(1-\delta^{d}\right)^{16}}
$$

Here

and

$$
\left(\frac{d}{d_{0}}\right)^{\frac{1}{2}-s} \leqslant\left(\frac{d}{d_{0}}\right)^{\frac{1}{2}} \leqslant\left(d-d_{0}+1\right)^{l} \leqslant 2^{\frac{1}{2}}\left(d-d_{0}\right)\left(d-d_{0}+1\right)
$$

$$
\left(1-\delta^{d}\right)^{16} \geqslant\left(1-\delta^{2}\right)^{16}
$$

On substituting these estimates in (14) we obtain

$$
\begin{aligned}
d_{0}^{8-1} \delta^{-d_{0}} \psi & \geqslant \frac{1}{4}\left(2^{\downarrow}-1\right)-2^{\frac{1}{1}}\left(1-\delta^{2}\right)^{-16} \sum_{k \geqslant 1} \frac{1}{2} k(k+1) \delta^{k} \\
& =\frac{1}{4}\left(2^{\downarrow}-1\right)-2^{\natural}\left(1-\delta^{2}\right)^{-16}(1-\delta)^{-3} \delta \\
& >0,
\end{aligned}
$$

since $\delta<40^{-1}$. This concludes the proof that $\psi>0$ and so of Lemma 2 .

5. Proof of Lemma 1. This lemma was already proved simply by Rankin for all $s>1$ (his Lemma 7). His proof does not naturally extend to $s \leqslant 1$. We may thus confine ourselves to the range

$$
0 \leqslant s \leqslant 1 \text {. }
$$

However, it would probably not be difficult to extend our proof to all $s \geqslant 0$.

On differentiating the identity (6) of $\S 2$ term by term with respect to $\log y$ we obtain

where

$$
\frac{1}{2} y \Gamma(s) \pi^{-s} G_{y}(x, y)=\theta(s)+\theta(1-s)+2 M,
$$

and

$$
\theta(s)=s(y / \pi)^{s} \Gamma(s) \xi(2 s)
$$

$$
\begin{aligned}
M= & y^{\frac{1}{t}} \sum_{r \geqslant 1} r^{s-\frac{1}{d}} \sigma_{1-2 s}(r) K_{s-\frac{1}{2}}(2 \pi r y) \cos 2 \pi r x \\
& +4 \pi y^{\frac{2}{2}} \sum_{r \geqslant 1} r^{s+\frac{1}{2}} \sigma_{1-2 s}(r) K_{s-1}(2 \pi r y) \cos 2 \pi r x .
\end{aligned}
$$

We shall show that $G_{y}(x, y)>0$ by showing that $\theta(s)+\theta(1-s)$ is fairly large and $M$ is fairly small in the range

$$
0 \leqslant s \leqslant 1, y \geqslant \frac{3}{2}
$$


under consideration. Most of the time we can estimate quite crudely.

We consider first $\theta(s)$ and write

$$
\eta=\frac{y}{\pi} \geqslant \frac{3}{2 \pi}
$$

Since $\theta(s)$ has a pole at $s=\frac{1}{2}$ with residue $\frac{1}{4} \eta^{\frac{1}{1}} \Gamma\left(\frac{1}{2}\right)=\frac{1}{4}(\eta \pi)^{\frac{1}{2}}$, it is convenient to treat

$$
\begin{aligned}
\theta^{*}(s) & =\theta(s)-\frac{(\eta \pi)^{\frac{1}{2}}}{2(2 s-1)} \\
& =\eta^{s} \Gamma(s+1) \zeta(2 s)-\left[(\eta \pi)^{\frac{1}{b}} / 2(2 s-1)\right] .
\end{aligned}
$$

Clearly

$$
\theta^{*}(s)+\theta^{*}(1-s)=\theta(s)+\theta(1-s) .
$$

It is probably well-known that

$$
\zeta(t) \geqslant \frac{1}{t-1}+\frac{1}{2}=\frac{t+1}{2(t-1)}
$$

for $t \geqslant 0$. [For the identity

$$
\zeta(t)=\frac{1}{t-1}+\frac{1}{2}+t \sum_{n>0} \int_{0}^{\frac{1}{2}}\left\{\frac{1}{\left(n+\frac{1}{2}-u\right)^{t+1}}-\frac{1}{\left(n+\frac{1}{2}+u\right)^{t+1}}\right\} u d u,
$$

which is an immediate consequence of Euler's summation formula when $R t>1$, continues to hold by analytic continuation when $\mathcal{R} t \geqslant 0]$. By (2l) and (22),

$$
\theta^{*}(s) \geqslant \frac{(2 s+1) \eta^{8} \Gamma(s+1)-(\eta \pi)^{i}}{2(2 s-1)}
$$

We may now apply the mean-value theorem to

$$
f(s)=(2 s+1) \eta^{s} \Gamma(s+1)
$$

since

$$
f\left(\frac{1}{2}\right)=(\eta \pi)^{\frac{1}{2}} .
$$

Hence

Now

$$
\inf _{0 \leqslant s \leqslant 1} \theta^{*}(s) \geqslant \frac{1}{4} \inf _{0 \leqslant t \leqslant 1} f^{\prime}(t)
$$

$$
\frac{f^{\prime}(t)}{f(t)}=\frac{2}{2 t+1}+\frac{\Gamma^{\prime}}{\Gamma}(t+1)+\log \eta
$$

From the tables of $\Gamma^{\prime} / \Gamma$ (e.g. in Jahnke and Emde [5]) one readily sees that

$$
\frac{2}{2[(r+1) / 10]+1}+\frac{\Gamma^{\prime}}{\Gamma}\left(\frac{r}{10}+1\right) \geqslant 0.9
$$

for $r=0,1,2, \ldots, 9$ and so, by the monotonicity of $2 /(2 t+1)$ and $\Gamma^{\prime}(t+1) / \Gamma(t+1)$, we have

$$
\frac{2}{2 t+1}+\frac{\Gamma^{\prime}}{\Gamma}(t+1) \geqslant 0.9 \quad(0 \leqslant t \leqslant 1) \text {. }
$$

Further,

$$
\log \eta \geqslant \log \frac{3}{2 \pi} \geqslant-0 \cdot 75
$$

Hence

$$
f^{\prime}(t) \geqslant 0 \cdot 15 f(t) \quad(0 \leqslant t \leqslant 1)
$$


Further, $\Gamma(t+1) \geqslant 0 \cdot 8$ for $0 \leqslant t \leqslant 1$, and so

where

$$
\begin{aligned}
f(t) & \geqslant(0.8)(2 t+1) \eta^{t} \\
& \geqslant(0.8)(2 t+1) \eta_{0}^{t},
\end{aligned}
$$

$$
\eta_{0}=\frac{3}{2 \pi}
$$

Now $\log \left\{(2 t+1) \eta_{0}^{t}\right\}$ is convex in $0 \leqslant t \leqslant 1$ and takes the values 0 and $\log 3 \eta_{0}>0$ at the two ends of the range. Hence, by (28),

$$
f(t) \geqslant 0 \cdot 8
$$

To sum up, from (24), (27), (29) we have

$$
\theta^{*}(s) \geqslant \frac{1}{4}(0 \cdot 15)(0 \cdot 8)=0.03 \quad(0 \leqslant s \leqslant 1) .
$$

[From the signs of the coefficients of $\eta^{s}$ and $\eta^{t}$ in $(21)$, it is clear that for fixed $s$ in $0 \leqslant s \leqslant 1$ the function $\theta^{*}(s)$ increases when $y$ increases, provided that it is positive, so it would have been enough to consider $y=\frac{3}{2}$. The numerical evidence suggests that then $\theta^{*}(s)$ increases in $0 \leqslant s \leqslant 1$. If so, the 0.03 in (30) could be replaced by the value of $\theta^{*}(0)$ when $y=\frac{3}{2}$, namely $\frac{1}{2}\left(\frac{3}{2}\right)^{1}-\frac{1}{2} \fallingdotseq 0 \cdot 1124$. But the inequality $(30)$ is much more than we in fact need.] we have

We can now estimate $|M|$ using the techniques of $\S 3$ but more crudely. For $|\nu| \leqslant 1$

$$
\begin{aligned}
0 \leqslant K_{\nu}(u) & =\int_{0}^{\infty} e^{-u \cosh t} \cosh v t d t \\
& \leqslant \int_{0}^{\infty} e^{-u \cosh t} \cosh t \cosh v t d t \\
& =-K_{\nu}^{\prime}(u) \\
& \leqslant \int_{0}^{\infty} e^{-u \cosh t} \cosh ^{2} t d t . \ldots . . .
\end{aligned}
$$

On applying these inequalities to $M$ and observing that

$$
y^{\frac{1}{2}} \leqslant y^{\mathrm{d}}, \quad r^{s-1} \leqslant r^{s+\frac{1}{2}}, \quad|\cos 2 \pi v x| \leqslant 1,
$$

we obtain

$$
|M| \leqslant(4 \pi+1) y^{\frac{3}{2}} \int_{0}^{\infty} \Psi\left(\delta_{t}\right) \cosh ^{2} t d t
$$

where

$$
\delta_{\ell}=e^{-2 \pi y \cosh t} \leqslant e^{-2 \pi y} \leqslant e^{-3 \pi} \leqslant 10^{-4},
$$

and $\Psi(\delta)$ is defined by replacing $\sin 2 \pi r x$ by 1 on the right-hand side of $(7)$ in $\S 4$. But now as in $\S 4$, we have

$$
|\Psi(\delta)|=\left|\sum_{d \geqslant 1} d^{\mathbb{l}-s} \Omega_{d}\right| \leqslant \sum_{d \geqslant 1} d^{\mathrm{n}}\left|\Omega_{d}\right|,
$$

where $\Omega_{d}$ is defined by replacing $\sin 2 \pi r x$ by 1 on the right-hand side of (10). The estimate $\left(10^{\prime}\right)$ holds with $\Omega_{d}$ instead of $\omega_{d}$. Hence by (33) and $\left(33^{\prime}\right)$,

$$
\begin{aligned}
|\Psi(\delta)| & \leqslant \sum_{d \geqslant 1} d \delta^{d}\left(1-\delta^{d}\right)^{-16} \leqslant(1-\delta)^{-20} \delta \\
& \leqslant(1 \cdot 1) \delta \ldots \ldots \ldots \ldots \ldots \ldots \ldots \ldots \ldots \ldots \ldots \ldots \ldots \ldots \ldots \ldots \ldots \ldots
\end{aligned}
$$


From (32) and (34), we have

$$
|M| \leqslant(4 \pi+1)(1 \cdot 1) y^{2} e^{-2 \pi y} I,
$$

where

$$
I=\int_{0}^{\infty} e^{-2 \pi y(\cosh t-1)} \cosh ^{2} t d t
$$

On making the substitution $v=\cosh t$ and observing that

one readily sees that

$$
e^{-2 \pi y(v-1)} \leqslant v^{-2 \pi y} \leqslant v^{-9},
$$

$$
I \leqslant \int_{1}^{\infty} \frac{v^{-7}}{\left(v^{2}-1\right)^{\frac{1}{1}}} d v \leqslant 1
$$

From (35) and (37) we have

$$
\begin{aligned}
& |M| \leqslant(4 \pi+1)(1 \cdot 1) y^{2} e^{-2 \pi y} \\
& <0.005 \text {, }
\end{aligned}
$$

since $y \geqslant \frac{3}{2}$. Thus finally, by (30) and (38),

$$
\begin{aligned}
\frac{1}{2} y \Gamma(s) \pi^{-s} G_{y}(x, y) & =\theta^{*}(s)+\theta^{*}(1-s)+2 M \\
& \geqslant 0.03+0.03-2(0.005) \\
& >0 .
\end{aligned}
$$

This concludes the proof of Lemma 1 and so of the theorem.

\section{REFERENCES}

1. R. A. Rankin, A minimum problem for the Epstein zeta-function, Prac. Glasgow Math. Assoc. 1 (1953), 149-158.

2. H. Weber, Lehrbuch der Algebra III, especially page 526 (Braunschweig, 2te Auflage, 1908).

3. M. Deuring, Zeta.funktionen quadratischer Formen, J. fur reine u. angew. Math. 172 (1935), 226-252.

4. G. N. Watson, A treatise on the theory of Bessel functions (Cambridge, 1922 (2nd ed. 1944)).

5. E. Jahnke and F. Emde, Funktionentafeln (Teubner, Leipzig (3rd ed. 1938)).

6. L. Kronecker, Ưber die Auflösung der Pell'schen Gleichung mittels elliptischer Functionen, Monatsber. d. Kön. Preuss. Akad. d. Wiss. zu Berlin 1863, 44-50 (=Werke IV, 219-226).

\section{Trinity College}

\section{Cambridge}

